\title{
Workspace Determination and Feedback Control of a Pick-and-Place Parallel Robot: Analysis and Experiments
}

\author{
Bruno Belzile, Peyman Karimi Eskandary and Jorge Angeles, Fellow, IEEE
}

\begin{abstract}
The authors report on the analysis and experimental evaluation of a parallel Schönflies-motion generator (SMG) intended for fast pick-and-place operations and actuated with cylindrical drives (C-drives). Its CRRHHRRC isostatic architecture offers high rotability of the moving platform and a reduced number of limbs, as compared to robots available on the market. A simulation model using the dynamics of the robot as well as an experimental prototype are described. The results obtained are used to assess the pros and cons of two alternative $C$ drive architectures. A workspace analysis including the feasible wrenches is conducted. The industry standard Adept test cycle is used to evaluate the performance of the prototype. The data obtained prove that $\mathrm{C}$-drives augmented with strain-wave gears give the SMG significantly better wrench capabilities and lower trajectory-tracking error, compared to their alternative $\mathrm{C}$-drives based on cables.
\end{abstract}

Index Terms-Parallel Robots, Schönflies Motion, SCARA robot, Dynamics, Force Control

\section{Motivation, Problem Statement, Related Work}

Pick-and-place operations (PPOs), which consist of grasping a payload from an original pose and moving it to a destination pose, have a broad range of industrial applications, including assembly and packaging tasks. The industry calls upon robots with high velocity while being capable of maneuverability and precision. Various serial and parallel architectures have been proposed over time to accomplish those tasks [1], [2], [3]. The former are known to offer a larger workspace and a longer reach than the latter, which can, on the other hand, lift heavier payloads. The best-known serial pick-and-place robot is the SCARA (Selective-Compliance Assembly Robot Arm), which has one prismatic and three revolute joints in a serial array [1].

Nowadays, most fast pick-and-place robots commercially available are parallel-kinematics machines (PKM). Among the parallel-serial and fully parallel architectures, the three-limb Delta architecture with a telescopic Cardan shaft [4] as well as the four-limb H4, I4L, I4R, Heli4 and Par4 [5] are well known. The Quattro $5650 H$, from Adept Technology Inc., is an example of the Par4 architecture, capable of three cycles per

Manuscript received: May, 22, 2019; Revised August, 30, 2019; Accepted September, 19, 2019

This paper was recommended for publication by Editor Dezhen Song upon evaluation of the Associate Editor and Reviewers' comments. This work was supported by NSERC (Canada's Natural Sciences and Engineering Research Council) through grant No. 4532-2010, and its Postdoctoral Fellowship Program and the McGill Engineering Doctoral Award.

The authors are with the Centre for Intelligent Machines and Department of Mechanical Engineering, McGill University bruno@cim.mcgill. ca

Digital Object Identifier (DOI): see top of this page. second. Because of the low-rotability of its moving platform by virtue of the four limbs, a complex mechanism is needed to amplify the rotation of the mobile platform (MP) [6]. A scarcity of parallel Schönflies-motion generators (SMGs) feature high-rotability; one of this class was proposed by Gosselin et al. [7]. The latter features a nonredundant parallel architecture with four coaxial revolute actuators, allowing infinite tool rotation on a large workspace. This concept, however, was not validated with a prototype.

Multiple approaches have been taken by different designers to improve the performance of various pick-and-place robots. For example, Clavel and Vischer worked on the kinematic calibration of a Delta robot, using parametric methods to improve by a factor of 15 its positioning accuracy [8]. Control also has a significant impact on the performance of a robot designed for fast PPO. Indeed, several researchers have worked on trajectory-planning with pick-and-place robots, considering different criteria to obtain optimal parameters. For example, Gasparetto et al. [9] proposed what they call a minimum-timejerk trajectory-planning algorithm. To this end, they minimized the rms value of joint jerk Euclidian norm along the whole trajectory. Otherwise, without modifying the path, Pallicciari et al. proposed a method to determine an energy-optimal trajectory by means of time-scaling [10]. In order to optimize the capabilities of their mechanisms, several researchers have analyzed their workspace [7], [11], [12]. Moreover, some have also looked into the wrench capabilities at the end-effector [13], [14], manipulability [15], [16], and kinematic sensitivity [17].

Our letter focuses on the evaluation of one particular parallel pick-and-place robot, dubbed the Peppermill Carrier ${ }^{1}$ (PMC). The PMC is an isostatic SMG based on the $\underline{C R R H H R R \underline{C}^{2}}$ closed kinematic chain [18]. It is noteworthy that the kinematic chains of most commercial SMGs, designed with only revolute and prismatic joints, for exactly the four degrees of freedom of the Schönflies-motions, are kinetostatically overconstrained. As a consequence, they cannot be assembled due to unavoidable manufacturing errors [19]. To cope with this issue, designers add extra joints to their robots, while keeping the capability of Schönflies-motion generation. However, extra joints add play and compliance to the structure, thereby compromising performance.

\footnotetext{
${ }^{1}$ The central part is dubbed the Peppermill, because of its similarities, both formal and functional, to the long Italian peppermill.

${ }^{2} \mathrm{R}, \mathrm{H}$ and $\mathrm{C}$ stand for revolute, helical (screw) and cylindrical joint, correspondingly, underlined characters denoting actuated joints.
} 
The architectures of the Quattro and the FlexPicker are derived from that of the Delta. The $\Pi$-joints of the Delta, using Hervé's terminology [20], were designed by Clavel using established practice in the art when designing a planar four-bar linkage, i.e., the above-mentioned П-joint. A typical four-bar linkage is usually driven by one actuated joint, the other three being passive. In the case of the $\Pi$ quadrilateron, all four joints are passive, and hence, realized as orientable $\mathrm{R}$ (revolute) joints, given that these allow for accommodation of manufacturing and assembly errors. Kinematically, such joints are equivalent to spherical joints, which bear two extra degrees of freedom, equivalent to two extra $\mathrm{R}$ joints. Each parallelogram mechanism (П-joint) of the Delta thus carries altogether eight "superfluous" joints, the quotation marks indicating that the qualifier applies only to the ideal linkage, not considering any fabrication errors. Moreover, the extra joints add to the play and the compliance of the overall parallelogram. The Quattro carries four such parallelograms, and hence, $8 \times 4=32$ extra passive joints. The FlexPicker carries only three parallelograms, and hence, only $8 \times 3=24$ extra joints. The PMC is one of the four isostatic SMG architectures proposed by Lee and Lee [18], [21], all of them having only two limbs. Therefore, the PMC does not have the drawbacks of the statically indeterminate kinematic chains of the well-known examples mentioned earlier. The Lee architectures feature a functionally symmetric, self-collision-free, single-loop layout. The PMC is actuated by two cylindrical drives (C-drives) that were first proposed by Harada et al. [22]. The C-drives, by virtue of a cylindrical differential mechanism, are capable of generating translational and rotational motion independently, with the former along a direction parallel to the axis of the latter. The PMC, as well as the other architectures proposed by the Lees [18], [21], does not have the rotability issue of the architectures mentioned above; the PMC offers high rotatability thanks to a gripper-rotating mechanism based on two coaxial screws.

In this letter, a description of the PMC, along with its kinematics and dynamics, are reported. The workspace and the feasible wrenches are also analyzed. Two different $\mathrm{C}$-drive architectures are tested on the PMC, one cable-driven $\left(\mathrm{C}_{\mathrm{C}}\right.$-drive $)$, one $\mathrm{SWG}^{3}$-driven $\left(\mathrm{C}_{\mathrm{SWG}}\right.$-drive $)$. Simulation results based on a dynamics model are reported. Experimental data are also provided and discussed. Finally, some practical insights are given, for further development.

\section{PEPPERMILL CARRIER}

\section{A. Kinematics}

The two C-drive architectures are tested in conjunction with the PMC. These two mechanisms were initially designed to overcome issues related to bending moments on the rotary coupling in the architecture proposed by Harada et al. [22]. The first, a cable-driven architecture, as shown in Fig. 1, is tested. The second, a strain-wave gear (SWG) speed reducer, shown in Fig. 2, is used as a replacement of the cable-driven mechanism. Kinematically, both alternatives are equivalent.

\footnotetext{
${ }^{3}$ strain-wave gear
}

The main difference resides in the high speed-reduction ratio $G$ in the $\mathrm{C}_{\mathrm{SWG}}$-drive ${ }^{4}$. These two versions of the $\mathrm{C}$-drive were proposed in an earlier paper [23] and tested with an external load generated by a mass of $0.5 \mathrm{~kg}$. In this letter, each drive is mounted on the PMC to compare their performance w.r.t. PPOs.

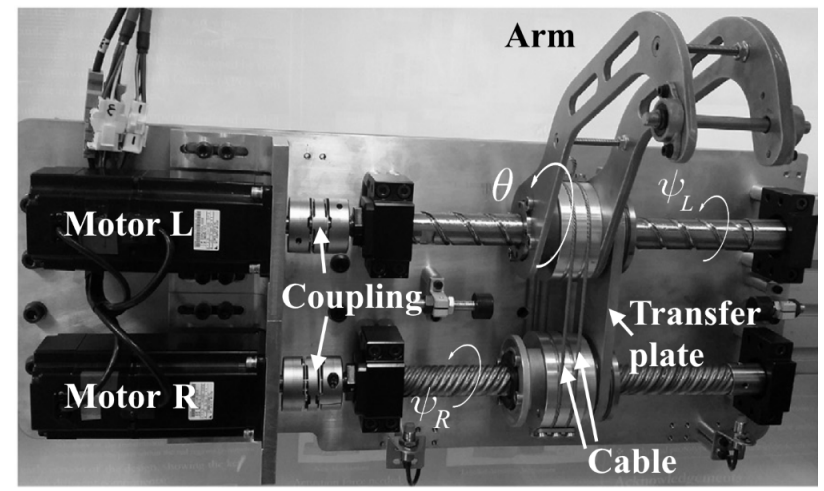

Fig. 1: C-drive supplied with a cable mechanism (for scale, ballscrews are $60 \mathrm{~mm}$ long)

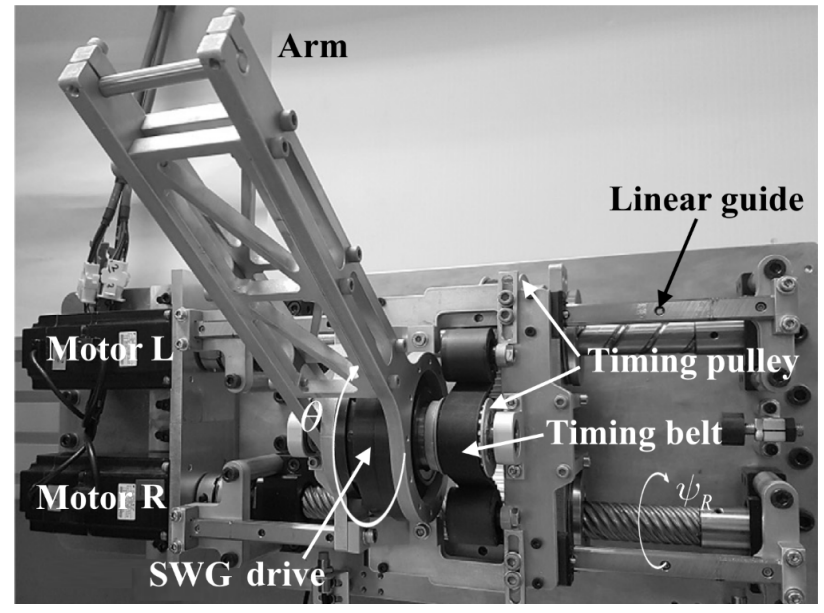

Fig. 2: C-drive supplied with a SWG (for scale, ballscrews are $60 \mathrm{~mm}$ long)

The complete kinematic chain of the PMC is illustrated in Fig. 3. The relationships between the homogeneous variables defining the displacement array $\boldsymbol{\sigma}$ of the collar of each Cdrive, and the angular-displacement array $\psi$ of the actuators are expressed via the "Jacobian"5 matrix $\mathbf{J}_{h}$ :

$$
\boldsymbol{\sigma}=\mathbf{J}_{h} \psi
$$

with

$$
\boldsymbol{\sigma}=\left[\begin{array}{c}
\sigma_{1} \\
\sigma_{2} \\
\sigma_{3} \\
\sigma_{4}
\end{array}\right]=\left[\begin{array}{c}
u_{1} \\
G p \theta_{1} /(2 \pi) \\
u_{2} \\
G p \theta_{2} /(2 \pi)
\end{array}\right] \text { and } \boldsymbol{\psi}=\left[\begin{array}{c}
\psi_{1 L} \\
\psi_{1 R} \\
\psi_{2 L} \\
\psi_{2 R}
\end{array}\right]
$$

\footnotetext{
${ }^{4}$ The SWG we used features a speed reduction of 22.5:1.

${ }^{5}$ The quotation marks indicate that the putative "Jacobian" is, actually, a constant, posture-independent coefficient matrix.
} 


$$
\mathbf{J}_{h}=\frac{p}{4 \pi}\left[\begin{array}{cccc}
1 & -1 & 0 & 0 \\
1 & 1 & 0 & 0 \\
0 & 0 & 1 & -1 \\
0 & 0 & 1 & 1
\end{array}\right]
$$

where $u_{i}$ and $\theta_{i}$ are the position and angle of the $i^{\text {th }} \mathrm{C}$ drive arm, $p$ is the pitch of the $\mathrm{C}$-drive screws, one right-, one left-hand, and $G$ is the gear reduction ratio of the Cdrive ( 1 for the cable-driven mechanism, 22.5 for its SWGdriven version). The subscripts $i L$ and $i R$ refer, respectively, to the motor coupled to the left-hand screw and right-hand screw of the $i^{\text {th }} \mathrm{C}$-drive. The pitch of $\mathrm{C}$-drive ballscrews is $60 \mathrm{~mm}$. Originally, these motors were also physically located at each end of the C-drive [22]. The Jacobian matrix $\mathbf{J}_{h}$ is dimensionally homogenous, with units of $\mathrm{m}$. The pose of the "moving platform", the gripper in Fig. 3, is defined by the vector $\mathbf{x}=\left[x_{c} y_{c} z_{c} \phi\right]^{T}$, whose components can be used to compute $\sigma$ :

$$
\left[\begin{array}{c}
\sigma_{1} \\
\sigma_{2} \\
\sigma_{3} \\
\sigma_{4}
\end{array}\right]=\left[\begin{array}{c}
x_{c} \\
\left(\alpha_{1}+\beta_{1}\right) G p /(2 \pi) \\
y_{c} \\
\left(2 \pi-\alpha_{2}-\beta_{2}\right) G p /(2 \pi)
\end{array}\right]
$$

with

$$
\begin{gathered}
\alpha_{1} \equiv \arctan \left(h_{1} / y_{c}\right), \quad \alpha_{2} \equiv \arctan \left(h_{2} / x_{c}\right) \\
\beta_{i} \equiv \arccos \frac{r^{2}-l^{2}-k_{i}^{2}}{2 r k_{i}} \\
k_{1} \equiv \sqrt{y_{c}^{2}+h_{1}^{2}}, \quad k_{2} \equiv \sqrt{x_{c}^{2}+h_{2}^{2}} \\
h_{i} \equiv z_{i}(-1)^{i} q \phi /(2 \pi)
\end{gathered}
$$

The geometric parameters are illustrated in Fig. 3.

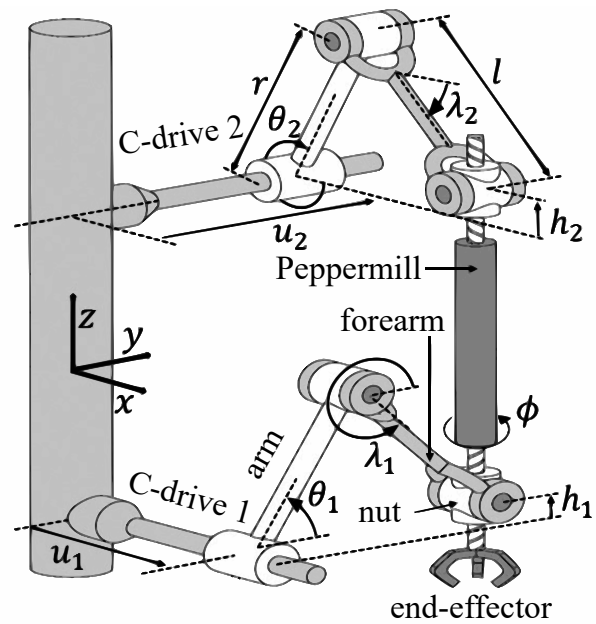

Fig. 3: PMC mechanism, a CRRHHRRC kinematic chain

\footnotetext{
${ }^{6}$ The quotation marks indicate that what plays the role of the moving platform in a PKM is, in fact, a rod-like link, as per Fig. 3
}

\section{B. Dynamics}

The inverse-dynamics model of the PMC, as reported elsewhere $^{7}$ [24], is used to determine the torques required from the actuators, a key issue in the design of the $\mathrm{C}$-drives. This model was obtained by means of the natural orthogonal complement [25]. For completeness, the inverse-dynamics model is briefly recalled below:

$$
\tau=\mathbf{I} \ddot{\psi}+\mathbf{C} \dot{\psi}-\gamma-\boldsymbol{\eta}-\boldsymbol{\delta}
$$

where

$$
\begin{aligned}
& \boldsymbol{\tau} \equiv\left[\begin{array}{llll}
\tau_{1 L} & \tau_{1 R} & \tau_{2 L} & \tau_{2 R}
\end{array}\right]^{T}, \mathbf{I} \equiv \sum_{i=1}^{11} \mathbf{T}_{i}^{T} \mathbf{M}_{i} \mathbf{T}_{i} \\
& \mathbf{C} \equiv \sum_{i=1}^{11}\left(\mathbf{T}_{i}^{T} \mathbf{M}_{i} \dot{\mathbf{T}}_{i}+\mathbf{T}_{i}^{T} \mathbf{W}_{i} \mathbf{M}_{i} \mathbf{T}_{i}\right) \\
& \boldsymbol{\gamma}+\boldsymbol{\eta}+\boldsymbol{\delta} \equiv \sum_{i=1}^{11} \mathbf{T}_{i}^{T}\left(\mathbf{w}_{i}^{G}+\mathbf{w}_{i}^{E}+\mathbf{w}_{i}^{D}\right) \\
& \mathbf{M}_{i} \equiv\left[\begin{array}{cc}
\mathbf{I}_{i} & \mathbf{O} \\
\mathbf{O} & m_{i} \mathbf{1}
\end{array}\right], \mathbf{W}_{i} \equiv\left[\begin{array}{cc}
\boldsymbol{\Omega}_{i} & \mathbf{O} \\
\mathbf{O} & \mathbf{O}
\end{array}\right], \quad \mathbf{T}_{i} \equiv \frac{\partial \mathbf{t}_{i}}{\partial \dot{\boldsymbol{\psi}}}
\end{aligned}
$$

Matrices $\mathbf{I}_{i}, \mathbf{M}_{i}, \mathbf{1}$ and $\mathbf{O}$ denote, respectively: the inertia tensor, the inertia dyad, both at the c.o.m. of the ith body; the $3 \times 3$ identity matrix; and the $3 \times 3$ zero matrix. The parameter $m_{i}$ is the mass of the $i$ th body. The angular-velocity dyad $\mathbf{W}_{i}$ of the $i$ th body is defined based on the angularvelocity matrix $\Omega_{i}$ of the $i$ th body, which is the cross-product matrix (CPM) of the angular-velocity vector $\boldsymbol{\omega}_{i}$. Moreover, $\mathbf{T}_{i}$, the twist-shaping matrix of the $i$ th body, maps the vector array of the motor speeds, the entries of array $\dot{\psi}$, into the twist vector $\mathbf{t}_{i}$ of the $i$ th body. Finally, $\mathbf{w}_{i}^{G}, \mathbf{w}_{i}^{E}$ and $\mathbf{w}_{i}^{D}$ denote, respectively, the gravitational, external and dissipative wrenches exerted on the $i$ th body; correspondingly, $\gamma, \boldsymbol{\eta}$ and $\delta$ are the generalized-force vectors, stemming from gravity, external loads, and dissipation. The twist $\mathbf{t}_{i}$ and the wrench $\mathbf{w}_{i}$, again evaluated at the body c.o.m., are defined as

$$
\mathbf{t}_{i} \equiv\left[\begin{array}{ll}
\boldsymbol{\omega}_{i}^{T} & \mathbf{v}_{i}^{T}
\end{array}\right]^{T}, \quad \mathbf{w}_{i} \equiv\left[\begin{array}{ll}
\mathbf{n}_{i}^{T} & \mathbf{f}_{i}^{T}
\end{array}\right]^{T}
$$

where $\mathbf{v}_{i}$ and $\boldsymbol{\omega}_{i}$ are, respectively, the velocity of $i$ th body c.o.m and the angular velocity of the same body, while $\mathbf{n}_{i}$ and $\mathbf{f}_{i}$ are the moment w.r.t. and the force acting on the $i$ th body, the latter at its c.o.m.. Preliminary experimental data, reported elsewhere [24], validated the inverse-dynamics model, showing that friction had a negligible effect on the torque calculations.

\section{Gear reduction ratio}

As already mentioned, the $\mathrm{C}_{\mathrm{SWG}}$-drive has a 22.5:1 gear reduction ratio. This value is not arbitrarily chosen, as it is optimum for the operation of the PMC. With the model presented above, the maximum absolute torque at any motor,

\footnotetext{
${ }^{7}$ The model can be found here: http://www.cim.mcgill.ca/ rmsl/Index/research.htm
} 
$\tau_{\max }(t) \equiv \max \left\{\left|\tau_{1 L}\right|,\left|\tau_{1 R}\right|,\left|\tau_{2 L}\right|,\left|\tau_{2 R}\right|\right\}$, was obtained as a function of the gear reduction ratio, the operation frequency for the Adept test cycle and time. Only the maximum value reached by $\tau_{\max }(t)$ along the trajectory is shown in Fig. 4. It should be noted that a logarithmic scale is used and that the inertia of the gear reduction mechanism is not taken into account.

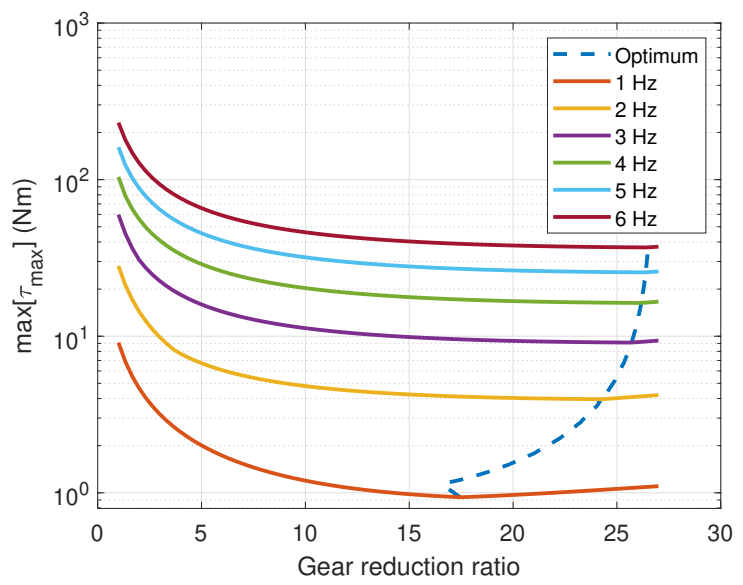

Fig. 4: Optimum gear reduction ratio

As can be observed, the maximum value of $\tau_{\max }(t)$ reaches a minimum between gear reduction ratios $10: 1$ and $30: 1$, depending on the operation frequency. For a frequency of 4 $\mathrm{Hz}$, which is the ultimate objective of the development of the current version of the PMC, the minimum is achieved with a gear reduction ratio of 26:1. Beyond this point, illustrated by the dashed curve for a spectrum between 1 and $6 \mathrm{~Hz}$ in Fig. 4, the maximum torque is mostly caused by the translation of the C-drives. The slight increase afterwards is caused by the additional rotation of the ballscrews required to generate the rotation, while the mass that is translated is unchanged. Therefore, the gear-reduction ratio should be chosen so as to minimize the effect of the rotation on the maximum torque, as if the C-drives were only translating. A larger ratio will not help reducing the torque peaks. Considering strain-wave-gear mechanisms available off-the-shelf, and considering that for operations below $4 \mathrm{~Hz}$, a smaller ratio is optimum, the ratio of 22.5:1 was considered the best choice for the prototype in light of the maximum absolute torque required at the actuators.

\section{Workspace}

The $x$ and $y$ boundaries of the PMC workspace are defined by the length of the screws of the $\mathrm{C}$-drives. The workspace may be widen by simply replacing the ballscrews of the $\mathrm{C}$ drives by longer ones. The vertical translation as well as the rotation of the end-effector, on the other hand, are limited by the maximum value that could take $\theta_{i}$ before hitting a mechanical limit, and the $(x, y)$-position of the Peppermill. The reachable workspace is illustrated in Fig. 5.

The wrench capabilities at every point of the workspace can also be obtained. To this end, eqs. $(3 \& 4)$ defining the Jacobian matrix of the PMC are used. The resulting polyhedra

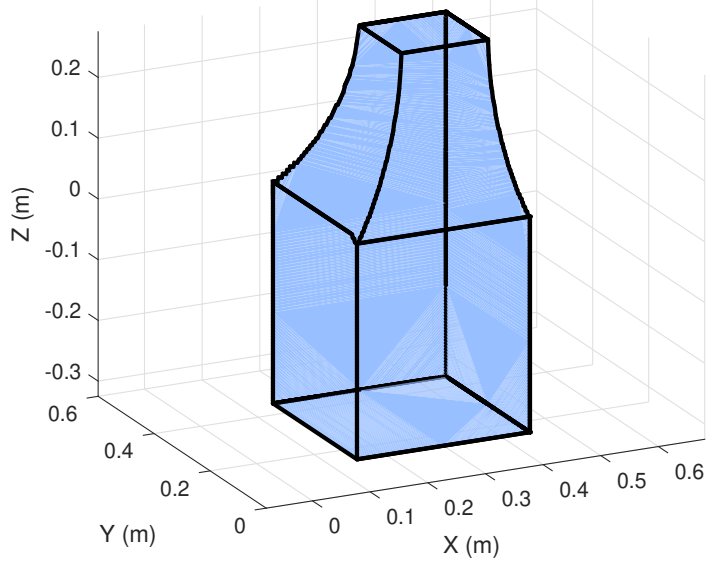

Fig. 5: Reachable workspace

are shown in Fig. 6, for the position $(0.300 \mathrm{~m}, 0.300 \mathrm{~m}$, $0.045 \mathrm{~m}$ ) in the PMC reference frame (without any rotation of the end-effector), for both the $\mathrm{C}_{\mathrm{C}}$-drives and the $\mathrm{C}_{\mathrm{SWG}^{-}}$ drives. As can be seen, the $\mathrm{C}_{\mathrm{SWG}}$-drives significantly improve the wrench capabilities of the PMC. The direction with the smallest maximum feasible wrench with $\mathrm{C}_{\mathrm{C}}$-drives is defined by the unit vector $[0.0345,-0.0285,0.9990]^{T}$. The magnitude of the corresponding force is $25.4 \mathrm{~N}$. With the $\mathrm{C}_{\mathrm{SWG}}$-drives, the smallest maximum force is defined by the unit vector $[-0.786,-0.0002,0.6181]^{T}$ and a magnitude of $329.3 \mathrm{~N}$. These numbers show that the wrench capabilities of the PMC with $\mathrm{C}_{\mathrm{C}}$-drives are mostly limited in the vertical direction.

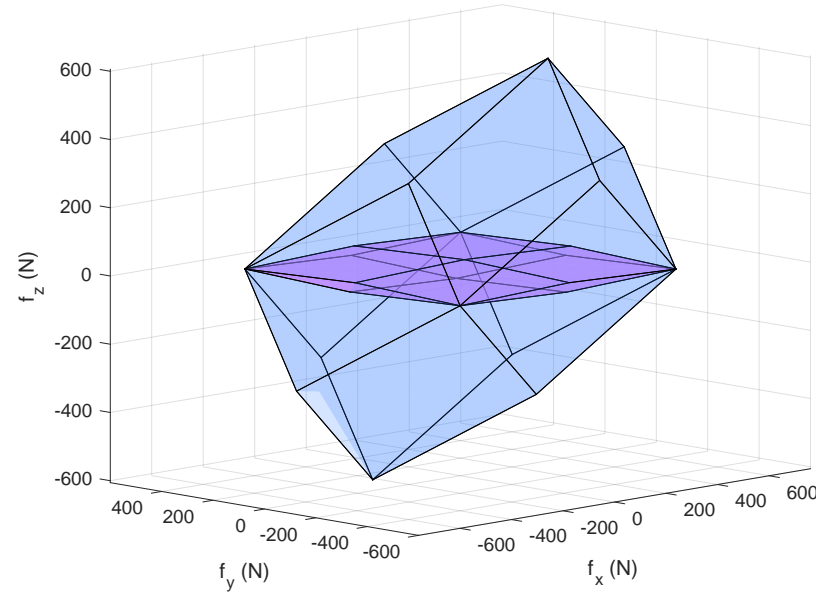

Fig. 6: Feasible wrenches (cable-driven, smaller purple polyhedron; SWG-based, larger blue polyhedron)

\section{E. Kinematic Sensitivity}

The local kinematic sensitivity is generally defined, at a given posture, as the measure of the effect of actuator displacements on the displacement of the MP. Metrics proposed by Cardou et al. [17] can be used to estimate the maximum rotation sensitivity and the maximum point-displacement sensitivity from the non-homogeneous Jacobian matrix of the 
robot. This is done by partitioning the Jacobian matrix into two blocks. For the PMC, the joint velocities are computed from the MP generalized velocities via the expression

$$
\dot{\psi}=\mathbf{J} \dot{\mathbf{x}}
$$

with

$$
\mathbf{J} \equiv\left[\begin{array}{ll}
\mathbf{K}_{p} & \mathbf{K}_{r}
\end{array}\right]=\left[\begin{array}{llll}
k_{p 11} & k_{p 12} & k_{p 13} & k_{r 11} \\
k_{p 21} & k_{p 22} & k_{p 23} & k_{r 21} \\
k_{p 31} & k_{p 32} & k_{p 33} & k_{r 31} \\
k_{p 41} & k_{p 42} & k_{p 43} & k_{r 41}
\end{array}\right]
$$

Blocks $\mathbf{K}_{p} \in \mathbb{R}^{4 \times 3}$ and $\mathbf{K}_{r} \in \mathbb{R}^{4 \times 1}$ relate, respectively, the Cartesian velocities of the MP to the angular velocities of the four motors located in the C-drives, and the MP angular velocity about the $Z$-axis to the same motor velocities. To be able to compute their kinematic sensitivity metrics, Cardou et $a l$. defined first a matrix $\mathbf{P}_{i}$, namely,

$$
\mathbf{P}_{i} \equiv \mathbf{1}_{4}-\mathbf{K}_{i}\left(\mathbf{K}_{i}^{T} \mathbf{K}_{i}\right)^{-1} \mathbf{K}_{i}^{T}, \quad i=p, r
$$

where $\mathbf{1}_{4}$ is the $4 \times 4$ identity matrix. The maximum pointdisplacement sensitivity $\sigma_{p}$ is defined as the inverse square root of the smallest eigenvalue of $\mathbf{K}_{p}^{T} \mathbf{P}_{r} \mathbf{K}_{p}$. Similarly, the maximum rotation sensitivity $\sigma_{r}$ is defined as the inverse square root of $\mathbf{K}_{r}^{T} \mathbf{P}_{p} \mathbf{K}_{r}$, since the latter is a scalar. The kinematic sensitivity for position in the $(x=y)$-plane are shown in Figs. 7 and 8. As can be seen, the values of these metrics are significantly smaller for the $\mathrm{C}_{\mathrm{SwG}}$-drive, which is better.

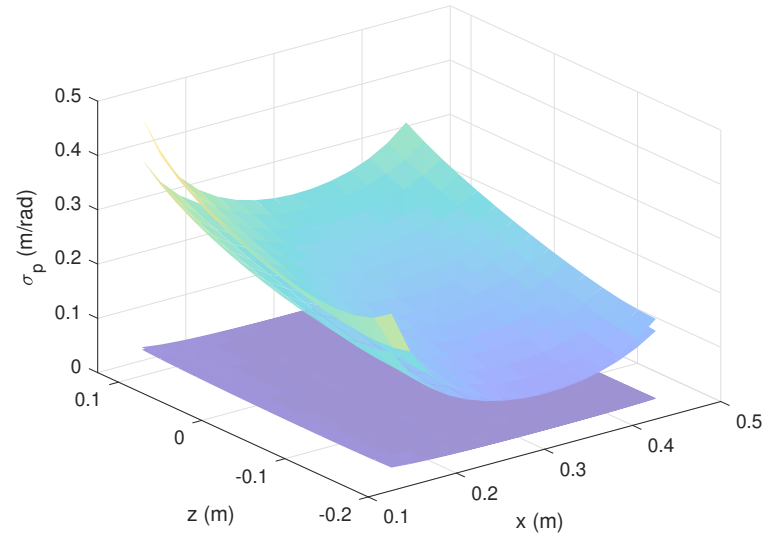

Fig. 7: Maximum point-displacement sensitivity: the two top surfaces correspond to the $\mathrm{C}_{\mathrm{C}}$-drives, showing the smallest and largest values of $\sigma_{p}$ in each position in the $(x=y)$ plane during a full rotation; the smallest and the largest values are indistinguishable in this figure for the $\mathrm{C}_{\mathrm{SwG}}$-drives, represented by the bottom surface.

\section{F. Prototype}

A picture of the Peppermill Carrier ${ }^{8}$ is shown in Fig. 9. The C-drives are actuated by two $750 \mathrm{~W}$ Yaskawa SGMAH

\footnotetext{
${ }^{8}$ Videos of the PMC in action can be found here: http://www.cim.mcgill.ca/ ^rmsl/Index/ research.htm
}

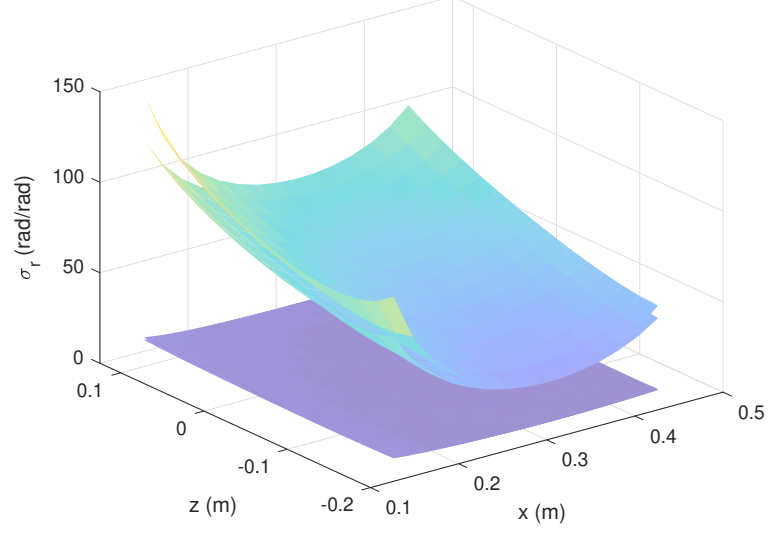

Fig. 8: Maximum rotation sensitivity: the two top surfaces correspond to the $\mathrm{C}_{\mathrm{C}}$-drives, showing the smallest and largest values of $\sigma_{p}$ in each position in the $(x=y)$-plane during a full rotation; the smallest and the largest values are indistinguishable in this figure for the $\mathrm{C}_{\mathrm{SWG}}$-drives, represented by the bottom surface

08AAF4C AC servomotors, rated at $2.39 \mathrm{Nm}$ and $3000 \mathrm{rpm}$. To provide feedback, 13-bit incremental shaft encoders are coupled to each motor. Four Yaskawa SGDH 08AE-S servomotor amplifiers are used, one for each motor. The RT-LAB platform is used to control the PMC and collect data from the sensors. The control scheme runs at $2 \mathrm{kHz}$. The arms and forearms are machined in aluminium. The end-effector is an electromagnet. The Peppermill is based on the virtual screw concept introduced earlier[26]. This mechanism is equivalent to the HH kinematic chain, but with cables and pulleys to be able to ajust the pitch of the virtual screws.

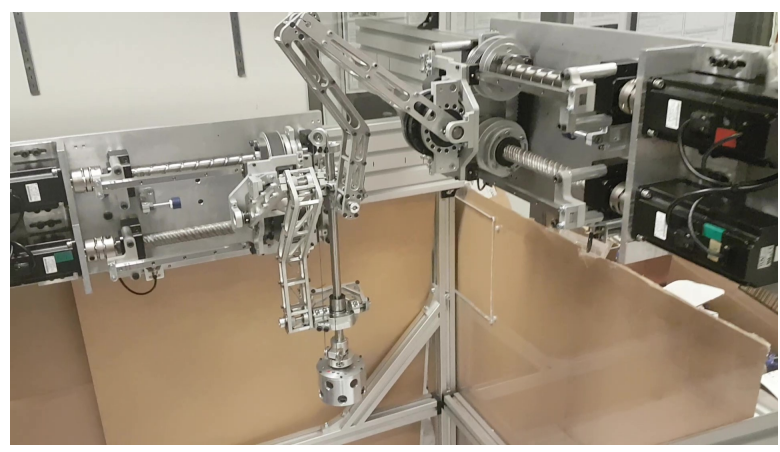

Fig. 9: Peppermill Carrier

\section{TRAJECTORY-TRACKING AND CONTROL}

The C-drives are controlled with a decentralized scheduledPID control scheme. The position and orientation of each Cdrive are controlled independently, each with its own controller, both controllers being identical. The control scheme described in this letter is different from an earlier version [27], which is based on a linear quadratic regulator. To test and assess the performance of SMGs, the industry-adopted test cycle [28]— the Adept test cycle—is used here. The latter 
consists of a vertical upward translation of $25 \mathrm{~mm}$, followed by a horizontal $300-\mathrm{mm}$ translation with a concurrent $180^{\circ}$ rotation about the vertical axis, then a vertical downward 25$\mathrm{mm}$ translation, and, finally, a motion back to the original gripper pose following the trajectory backwards. The trajectory was smoothed to avoid discontinuities in the time-domain [29].

The complete control scheme is shown in Fig. 10. Usually, the derivative part of the controller is filtered, to cope with the noise caused by a direct differentiation. The references for the controller are the homogeneous translational and angular positions of each $\mathrm{C}$-drive ( $\sigma_{1}$ and $\sigma_{2}$ for the first $\mathrm{C}$-drive, $\sigma_{3}$ and $\sigma_{4}$ for the second), which can be computed from the four gripper-pose variables $\left(x_{r}, y_{r}, z_{r}, \phi_{r}\right)$ defining the trajectory. The subscript $r$ indicates the reference trajectory. The inverse of the Jacobian matrix $\mathbf{J}_{h}$ is then used to compute the torques to be generated by the two motors of each $\mathrm{C}$-drive. It should be noted that the matrix $\mathbf{J}_{h}^{*}$ in Fig. 10 stands for the upper left $2 \times 2$ block of $\mathbf{J}_{h}$, since the two C-drives are controlled independently.

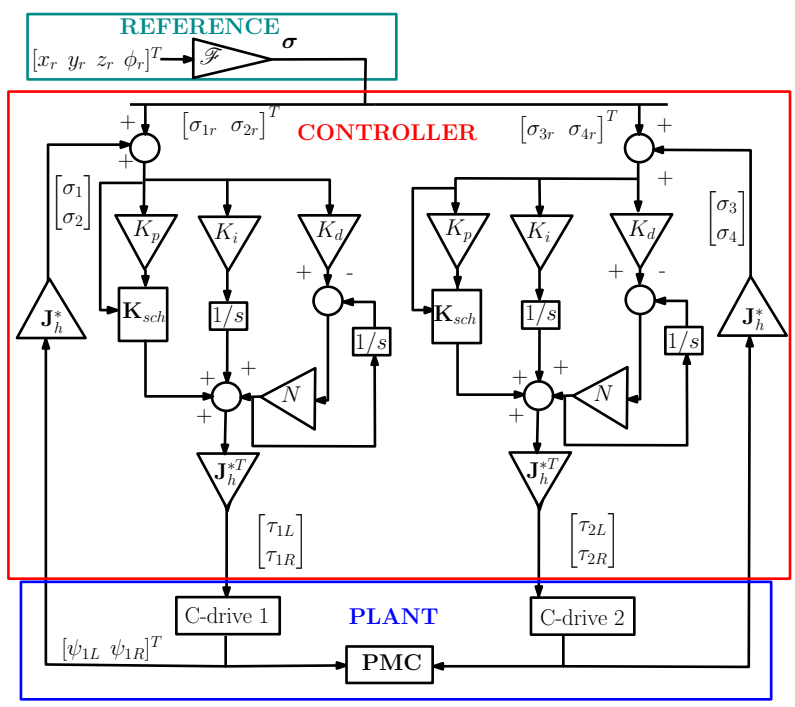

Fig. 10: PMC control scheme

Constants $K_{p}, K_{i}$ and $K_{d}$ are, respectively, the proportional, integral and derivative gains of the PID controller. The gain matrix $\mathbf{K}_{s c h} \equiv \operatorname{diag}\left(K_{u}, K_{v}\right)$, is a function of the error. The relationship between the latter and the gains $K_{u}$ and $K_{v}$ is shown in Fig. 11. Those scheduled gains are introduced to avoid oscillations near the reference pose, which were first observed during the preliminary tests with a standard PID controller.

\section{Simulation TESTS}

A simulation testbed was built with the model developed in Section II to analyze the behavior of the PMC and to validate the controller parameters. The angular acceleration of each motor for an Adept test cycle conducted at $2 \mathrm{~Hz}$ is shown in Fig. 12. Those results are obtained with two $\mathrm{C}_{\mathrm{C}}$-drives. Notice that the symmetry of both the robot architecture and the trajectory is reflected in the plots in this figure.

In comparison with the SWG version of the C-drives, whose acceleration plots are illustrated in Fig. 13, one can see that the

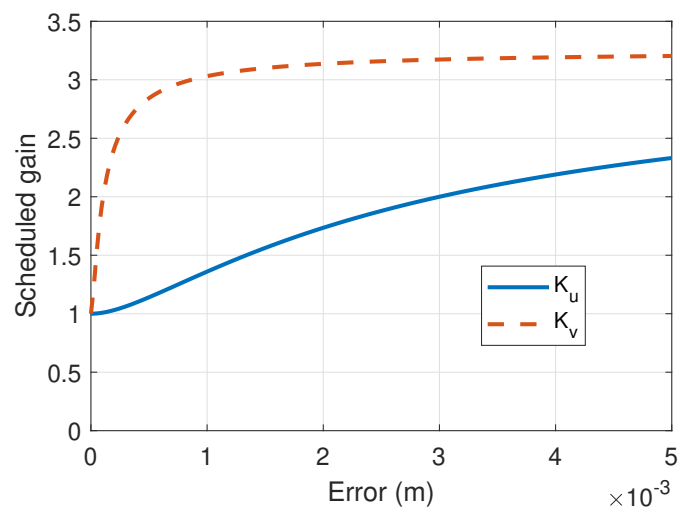

Fig. 11: Scheduled gains

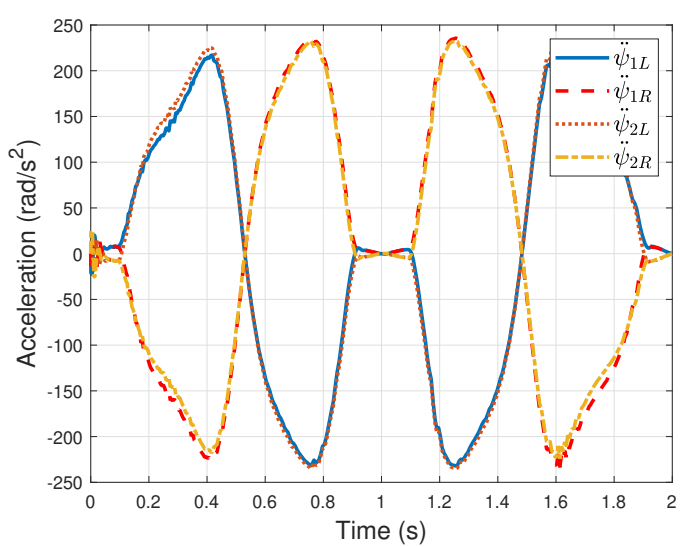

Fig. 12: Acceleration at each actuator along the prescribed trajectory with the $\mathrm{C}_{\mathrm{C}}$-drive $(1 \mathrm{~L}$ : 1 st $\mathrm{C}$-drive left motor; $1 \mathrm{R}$ : 1st C-drive right motor; 2L: 2nd C-drive left motor; 2R: 2nd C-drive right motor)

accelerations required with the $\mathrm{C}_{\mathrm{C}}$-drives are globally lower.

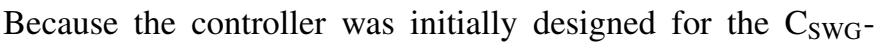
drive, some noise is visible in Fig. 12. The gains were not modified for the second set of C-drives for the sake of comparison. It is also noteworthy that the angular acceleration plots of the two motors of each $\mathrm{C}_{\mathrm{SWG}}$-drive are nearly symmetric with the cable-driven mechanism. Fully symmetric curves $(i L$ vs $i R$ ) would mean that the $\mathrm{C}$-drive is acting as a prismatic joint without any rotation. Thus, the small difference in Fig. 12 is caused by the rotational component $\theta_{i}$ of the cylindrical motion. Therefore, most of the torque required with the $\mathrm{C}_{\mathrm{C}^{-}}$ drives is used for the translational motion, contrary to the $\mathrm{C}_{\text {SWG-drive. }}$

However, the maximum torque generated is lower with the $\mathrm{C}_{\mathrm{SWG}}$-drives, as the effect of gravity is reduced by a factor of 22.5 , thanks to the gear reduction provided by the strain-wave gear mechanism. This makes friction negligible when compared with the dynamics effects of the system. The maximum torque required, regardless of the actuator, reached at any moment of the trajectory, is shown in Fig. 14.

The results for the C-drive architectures are now discussed. These results are highly meaningful, because they show that 


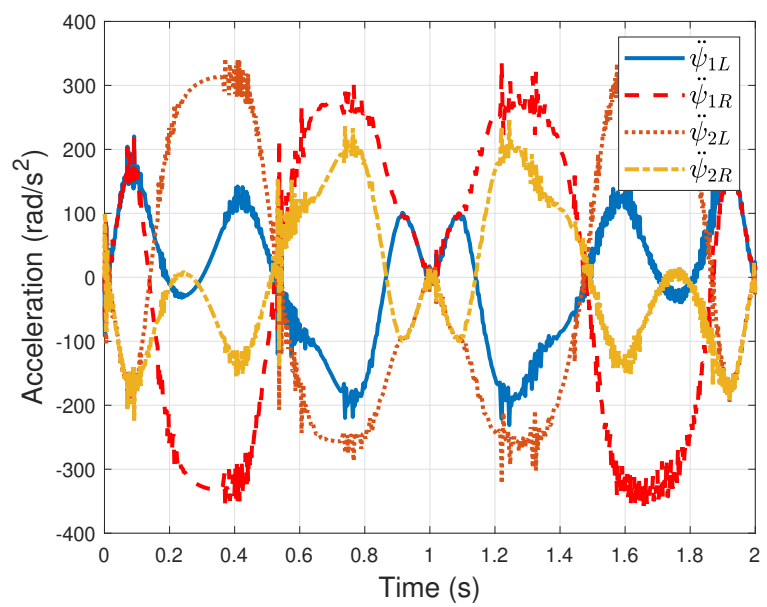

Fig. 13: Acceleration at each actuator along the prescribed trajectory with the $\mathrm{C}_{\mathrm{SWG}}$-drives

there is a significant amount of energy lost with the cablebased C-drives just to maintain the Peppermill at its rest pose. To solve this issue, a vertical force was applied by a static load to reduce the effect of gravity on the PMC when the $\mathrm{C}_{\mathrm{C}}$-drives were used, but this solution is not appropriate for high-speed operations. It should be noted that the vertical force generated by a counterweight is not required when the $\mathrm{C}_{\mathrm{SWG}}$-drives are mounted on the $\mathrm{PMC}$, making them more appropriate for higher operation frequencies.

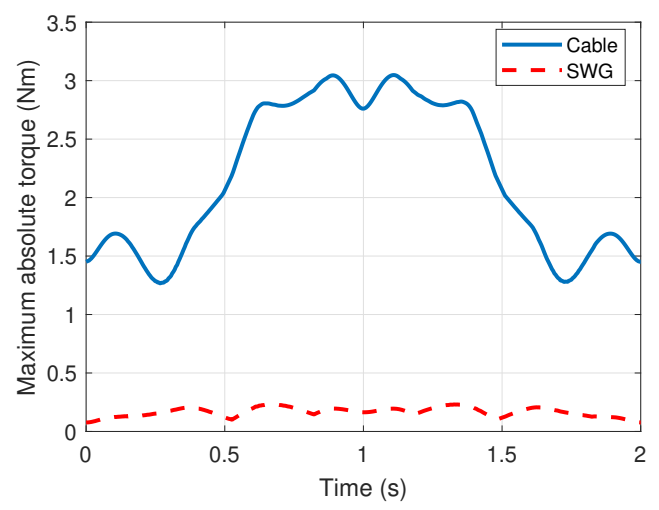

Fig. 14: Maximum absolute torque required at any motor at any point in the trajectory conducted at $0.5 \mathrm{~Hz}$

\section{EXPERIMENTAL EVALUATION}

Different experiments were conducted, again with the two versions of the C-drives. Initial tests were conducted with the $\mathrm{C}_{\mathrm{C}}$-drives, and the vertical force mentioned in Section IV. First and foremost, a large range of PID gains was tested. In Fig. 15, the trajectory-tracking at the C-drives can be seen with the best combination found of PID gains. The trajectory of the Adept cycle was followed at a rate of 0.5 cycles/s.

As observed, good trajectory-tracking is obtained in the $X$ and $Y$-translations, but there is a non-negligible error in the rotation. On the other hand, when the two $\mathrm{C}_{\mathrm{C}}$-drives were
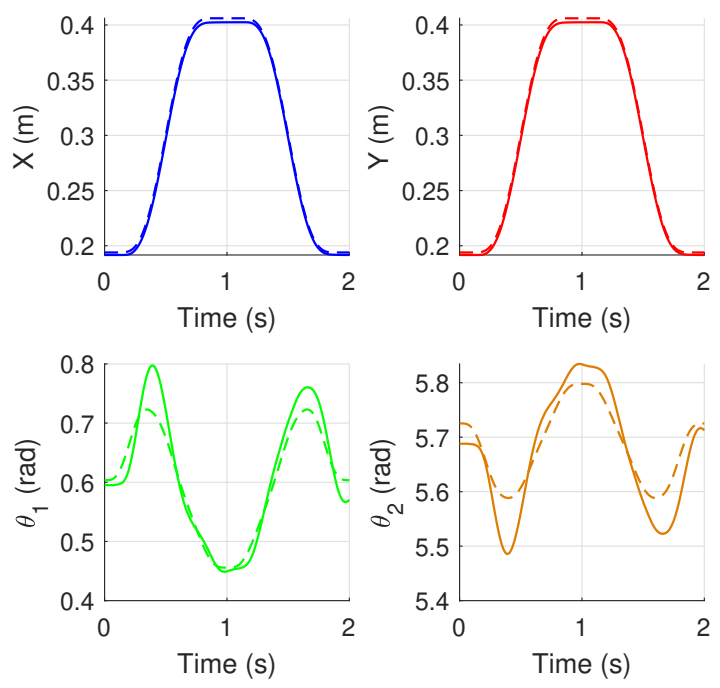

Fig. 15: Trajectory-tracking at the $\mathrm{C}_{\mathrm{C}}$-drives (reference: dashed curves; measured: solid curves) at a frequency of $0.5 \mathrm{~Hz}$, with the $\mathrm{C}_{\mathrm{C}}$-drives

replaced by their SWG-driven counterparts, good trajectorytracking was obtained in both translation and rotation. The same controller with the same gains, initially optimized for the $\mathrm{C}_{\mathrm{C}}$-drives, is used. The errors on the translation and the

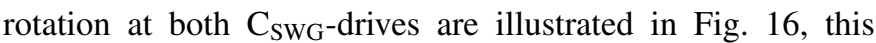
time with a higher frequency of $1.5 \mathrm{~Hz}$. For this figure, only the error is shown, as the reference and measured data would have been indistinguishable because of the scale. Whereas there could be an error of up to $0.1 \mathrm{rad}$ when using the $\mathrm{C}_{\mathrm{C}^{-}}$ drive, the maximum error observed is now $0.002 \mathrm{rad}$, i.e., an improvement by a factor of 50. This confirms that even at a higher velocity and following a more complex trajectory with a varying load, the $\mathrm{C}_{\mathrm{SWG}}$-drives still keep a highly good accuracy.

\section{Discussion}

Simulation and experimental tests with the $\mathrm{C}_{\mathrm{C}}$-drives revealed the limitations of the latter, namely the difficulty to precisely track an angular position of the C-drive at high speed, the resulting error being amplified at the MP, thereby causing large errors in $Z$ and $\phi$. Indeed, simulation results have shown that, naturally depending on the value $\theta_{i}$ of the angular position at the $\mathrm{C}$-drives, the absolute rotational error at the C-drives can be magnified 10 times at the MP of the PMC. Moreover, this estimation of the amplification of the error is obtained by considering a completely rigid mechanism, which is obviously not the case in practice. The $\mathrm{C}_{\mathrm{SWG}_{\mathrm{G}} \text {-drives }}$ solve this problem by reducing the error by a factor of 50 . Nearly identical results were obtained with more than 50 cycles performed.

Further work will include a stiffness analysis of the PMC. Considering the objective to increase the speed of the system, vibration at the moving platform will have to be studied. Indeed, a good trajectory-tracking at the C-drives would be useless if the error were amplified at the Peppermill pose. 

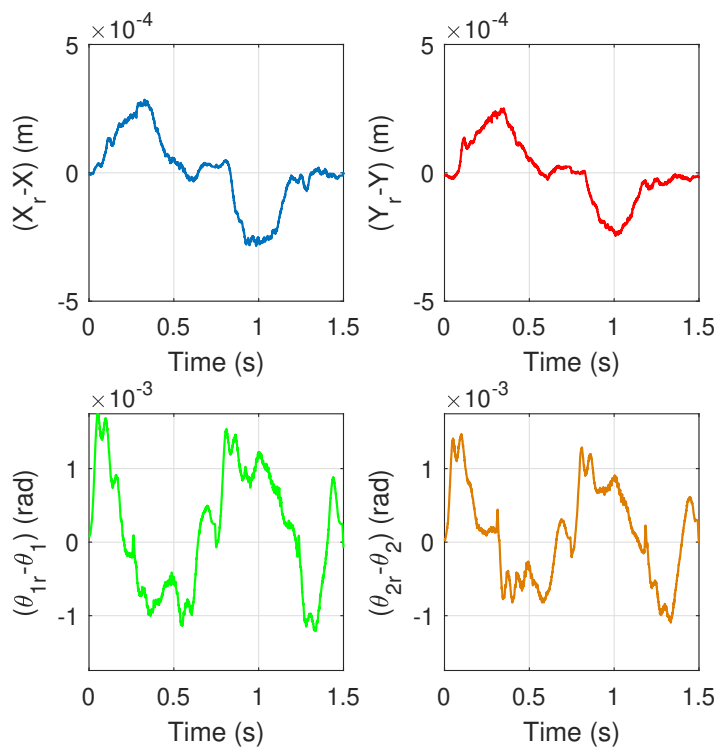

Fig. 16: Errors at the $\mathrm{C}_{\mathrm{SWG}}$-drives at a frequency of $0.667 \mathrm{~Hz}$

\section{CONCLUSIONS}

The dynamic response of the Peppermill Carrier, a $\underline{\text { CRRHHRRC }}$ parallel Schönflies-motion generator, was reported, along with a description of the physical realization of this kinematic chain, actuated by two identical cylindrical drives. The PMC is a work in progress. It is not (yet) as fast as the Quattro and the FlexPicker. However, its isostaticity and virtually unlimited rotability appear to be attractive features that should pave the way to the next generation of fast SMGs. A dynamics model was used to test the controller, a scheduled-PID scheme, and shed light on how the prototype can be improved to achieve higher speeds without reducing the trajectory-tracking accuracy. A workspace analysis was also included, and the wrench capabilities of the PMC were assessed. Experimental tests were conducted to corroborate the simulation results. The tests confirmed that the $\mathrm{C}_{\mathrm{SWG}}$-drive is an appropriate solution to the limited torque capability of the original $\mathrm{C}_{\mathrm{C}}$-drive.

\section{REFERENCES}

[1] H. Makino, A. Kato, and Y. Yamazaki, "Research and commercialization of SCARA robot-the case of industry-university joint research and development," International Journal of Automation Technology, vol. 1, pp. 61-67, 2007.

[2] C.C. Lee, and J.M. Hervé, "Type synthesis of primitive schönfliesmotion generators," Mechanism and Machine Theory, vol. 44, pp. 19801997, 2009

[3] S. Briot, and I.A. Bonev, "Pantopteron-4: A new 3T1R decoupled parallel manipulator for pick-and-place applications," Mech Mach Theory, vol. 45 , pp. 707-721, 2010.

[4] R. Clavel, "Device for the Movement and Positioning of an Element in Space," U.S. Patent No. 4,976,582, 1990.

[5] F. Pierrot, T. Shibukawa, and K. Morita, "Four-Degree-of-Freedom Parallel Robot," U.S. Patent No. 6,516,681, 2003.

[6] O. Company, F. Pierrot, S. Krut, and V. Nabat, "Simplified dynamic modelling and improvement of a four degree-of-freedom pick-andplace manipulator with articulated moving platform," Proceedings of the Institution of Mechanical Engineers, Part I: Journal of Systems and Control Engineering, vol. 223, no. 1, pp. 13-29, 2009.
[7] C. Gosselin, M. Isaksson, K. Marlow, and T. Laliberté, "Workspace and Sensitivity Analysis of a Novel Non-Redundant Parallel SCARA Robot Featuring Infinite Tool Rotation," IEEE Robotics and Automation Letters, vol. 1, no. 2, pp. 776-783, 2016.

[8] P. Vischer, and R. Clavel, "Kinematic calibration of the parallel Delta robot," Robotica, vol. 16, pp. 207-218, 1998.

[9] A. Gasparetto, A. Lanzutti, R. Vidoni, and V. Zanotto, "Validation of minimum time-jerk algorithms for trajectory planning of industrial robots," ASME Journal of Mechanisms and Robotics, vol. 3, no. 3, 031003, 2011.

[10] M. Pellicciari, G. Berselli, F. Leali, and A. Vergnano, "A method for reducing the energy consumption of pick-and-place industrial robots," Mechatronics, vol. 23, no. 3, pp. 326-334, 2013.

[11] A.L. Orekhov, C.B. Black, J. Till, S. Chung, and D.C. Rucker, "Analysis and Validation of a Teleoperated Surgical Parallel Continuum Manipulator," IEEE Robotics and Automation Letters, vol. 1, no. 2, pp. 828-835, 2016.

[12] A. Lotfavar, S. Hasanzadeh, and F. Janabi-Sharif, "Cooperative Continuum Robots: Concept, Modeling, and Workspace Analysis," IEEE Robotics and Automation Letters, vol. 3, no. 1, pp. 426-433, 2018.

[13] F. Firmani, A. Zibil, S. Nokleby, and R.P. Podhorodeski, "Wrench capabilities of planar parallel manipulators. Part I: Wrench polytopes and performance indices," Robotica, vol. 26, no. 6, pp. 791-802, 2008.

[14] L. Mejia, H. Simas, and D. Martins, "Wrench capability in redundant planar parallel manipulators with net degree of constraint equal to four, five or six," Mech Mach Theory, vol. 105, pp. 58-79, 2016.

[15] T. Yoshikawa, "Manipulability of robotic mechanisms," International Journal of Robotic Research, vol. 4, no. 2, pp. 3-9, 1985.

[16] J.-P. Merlet, "Jacobian, manipulability, condition number, and accuracy of parallel robots," ASME Journal of Mechanical Design, vol. 128, no. 1, pp. 199-206, 2006.

[17] P. Cardou, S. Bouchard, and C. Gosselin, "Kinematic-Sensitivity Indices for Dimensionally Nonhomogeneous Jacobian Matrices," IEEE Transactions on Robotics, vol. 26, no. 1, pp. 166-173, 2010.

[18] C.C. Lee, P.C. Lee, Y. Lou, and Z. Li, "Isoconstrained mechanisms for fast pick-and-place manipulation," in Geometric Methods in Robotics and Mechanism Research: Theory and Applications, Saarbrücken: LAP Lambert Academic Publishing, pp. 95-112, 1990.

[19] T. Zou, M. Shaker, J. Angeles, and A. Morozov, "An innovative tooth root profile for spur gears and its effect on service life," Meccanica, vol. 52 , pp. $1825-1841,2017$.

[20] J.M. Hervé, "The lie group of rigid body displacements, a fundamental tool for mechanism design," Mechanism and Machine Theory, vol. 34, pp. 719-730, 1999.

[21] P.C. Lee, and J.J. Lee, "Singularity and workspace analysis of three isoconstrained parallel manipulators with Schönflies motion," Frontiers of Mechanical Engineering, vol. 7, pp. 163-187, 2012.

[22] T. Harada, T. Friedlaender, and J. Angeles, "The development of an innovative two-dof cylindrical drive: Design, analysis and preliminary tests," in Proceedings of the IEEE International Conference on Robotics and Automation, Hong Kong, 2014.

[23] P. Karimi Eskandary, and J. Angeles, "The translating П-joint: Design and applications," Mech Mach Theory, vol. 122, pp. 361-370, 2018.

[24] P. Karimi Eskandary, and J. Angeles, "The dynamics of a parallel Schönflies-motion generator," Mech Mach Theory, vol. 119, pp. 119129, 2018.

[25] J. Angeles, and S. Lee, "The formulation of dynamical equations of holonomic mechanical systems using a natural orthogonal complement," ASME Journal of Applied Mechanics, vol. 55, pp. 243-244, 1988.

[26] P. Karimi Eskandary, and J. Angeles, "The virtual screw: Concept, design and applications," Mech Mach Theory, vol. 128, pp. 349-358, 2018.

[27] P. Karimi Eskandary, B. Belzile, and J. Angeles, "Trajectory-Planning and Normalized-Variable Control for Parallel Pick-and-Place Robots," ASME Journal of Mechanisms and Robotics, in press, 2019.

[28] V. Nabat, M. Rodriguez, O. Company, S. Krut, and F. Pierrot, "Par4: Very high speed parallel robot for pick-and-place," in Proceedings of the IEEE/RSJ International Conference on Intelligent Robots and Systems, Edmonton, Canada, 2005.

[29] J.F. Gauthier, J. Angeles, and S.B. Nokleby, "Optimization of a test trajectory for SCARA systems," in Lenarcic, J. Thomas, F. (Eds.), On Advances in Robot Kinematics, Amsterdam: Springer, 2008, pp. 225 234 